\title{
Polyclonal aptamer libraries as binding entities on a graphene FET based biosensor for the discrimination of apo- and holo- retinol binding protein 4
}

\author{
Ann-Kathrin Kissmann ${ }^{\ddagger[a]}$, Jakob Andersson ${ }^{\ddagger *[b]}$, Heinz Fabian Raber ${ }^{\ddagger[a]}$, Markus Krämer ${ }^{[a]}$, Hu Xing ${ }^{[a]}$, \\ Dennis H. Kubiczek ${ }^{[a]}$, Anil Bozdogan ${ }^{[c]}$, Patrik Aspermair ${ }^{[b]}$, Wolfgang Knol[ ${ }^{[b]}$ and Frank Rosenau ${ }^{* a][d]}$
}

[a] A.-K. Kissmann, Dr. H. F. Raber, M. Krämer, H. Xing, Dr. D. H. Kubiczek, Dr. F. Rosenau

Institute of Pharmaceutical Biotechnology

Ulm University, Albert-Einstein-Allee 11, 89081 Ulm, Germany

E-mail: frank.rosenau@uni-ulm.de

[b] Dr. J. Andersson, P. Aspermair, Prof. Dr. W. Knoll

Austrian Institute of Technology $\mathrm{GmbH}$

Giefinggasse 4, 1210 Vienna, Austria

E-mail: jakob.andersson@ait.ac.at

[c] A. Bozdogan

CEST Kompetenzzentrum für Elektrochemische Oberflächentechnologie GmbH

Viktor Kaplan Straße 2, Wiener Neustadt, Austria Institution

$\ddagger$ These authors contributed equally.

\begin{abstract}
Oligonucleotide DNA aptamers represent an emergently important class of binding entities towards as different analytes as small molecules or even whole cells. Without the canonical isolation of individual aptamers following the SELEX process already the focused polyclonal libraries prepared by this in vitro evolution and selection can directly be used to label their dedicated analytes and to serve as binding molecules on surfaces. Here we report the first instance of a sensor able to discriminate between loaded and unloaded retinol binding protein 4 (RBP4), an important biomarker for the prediction of diabetes and kidney disease. The sensor relies purely on two aptamer libraries tuned such that they discriminate between the protein isoforms, requiring no further sample labelling to detect RBP4 in both state. The evolution, binding properties of the libraries and the functionalization of graphene FET sensor chips are presented as well as the functionality of the resulting biosensor.
\end{abstract}

\section{Introduction}

Retinol binding protein 4 (RBP4) is a serum protein which belongs to the lipocalin ligand binding protein family. ${ }^{[1]}$ It serves as a transporter in the blood for alltrans-retinol (vitamin A alcohol) and other retinoids like retinal or retinoid acid from hepatic retinoid stores to peripheral tissues of the body. ${ }^{[2]}$ RBP4 is expressed by the liver and circulates in blood serum, ${ }^{[3]}$ where normal levels of the protein can range from 40 to $60 \mu \mathrm{g} / \mathrm{mL}$ in humans. ${ }^{[4]}$ Under physiological conditions, mainly ligand-bound RBP4 (holo-RBP4), approximately $90 \%$ of blood RBP4, ${ }^{[5]}$ circulates in an 1:1:1 complex with transthyretin (TTR) (Scheme 1) and all-trans-retinol as its stability is further enhanced and RBP4 is prevented from extensive loss throughout glomerular filtration and catabolism in the kidneys. ${ }^{[6,7]}$ Under healthy conditions, the RBP4 expression is tightly regulated and after the transport of retinol to its target cells the remaining unbound RBP4 (apo-RBP4) is rapidly filtered out and the protein is cleared from the serum. ${ }^{[8]}$ However, the amount of RBP4 can be increased in the serum as a consequence of diseases like obesity, ${ }^{\left[{ }^{[9]}\right.}$ chronic kidney disease, ${ }^{[10]}$ insulin resistance, ${ }^{[11]}$ and type 2 diabetes mellitus. ${ }^{[12]}$ Depending on the level of elevation of the concentration of a certain RBP4 isoform or disbalances of RBP4 to retinol ratios various reactions affecting the human health state can be triggered. ${ }^{[13]}$ Recent studies showed, that especially apo-RBP4 contributes in adipose tissue to the development of an inflammatory state, which may result in insulin resistance. ${ }^{[14,15]}$ Likewise, patients suffering from type 2 diabetes mellitus may exhibit moderate plasma RBP4 levels, but their level of free RBP4 in proportion to retinol is increased indicating an imbalance in ratios as a crucial factor. ${ }^{[16] ~ N o w a d a y s, ~ s e v e r a l ~ e n z y m e ~ l i n k e d ~}$ immunosorbent assays (ELISAs) are commercially available the for detection of RBP4 in human serum, but they lack accuracy and are insufficient for the quantification of high concentrations of RBP4. ${ }^{[17]}$ Hence, quantitative western blotting is used as the standard procedure for the detection of serum RBP4 especially in insulin-resistant states. ${ }^{[12]}$ There, normally only the amount of total RBP4 is measured and no further differentiation in isoforms is made. In order to overcome these restrictions and to create a methodology allowing to discriminate between apo- and holo-RBP4 the 
development of specific binding molecules with distinct affinities either for the apo- or the holo -protein would be an attractive amendment of the current existing RBP4 specific diagnostic technologies.

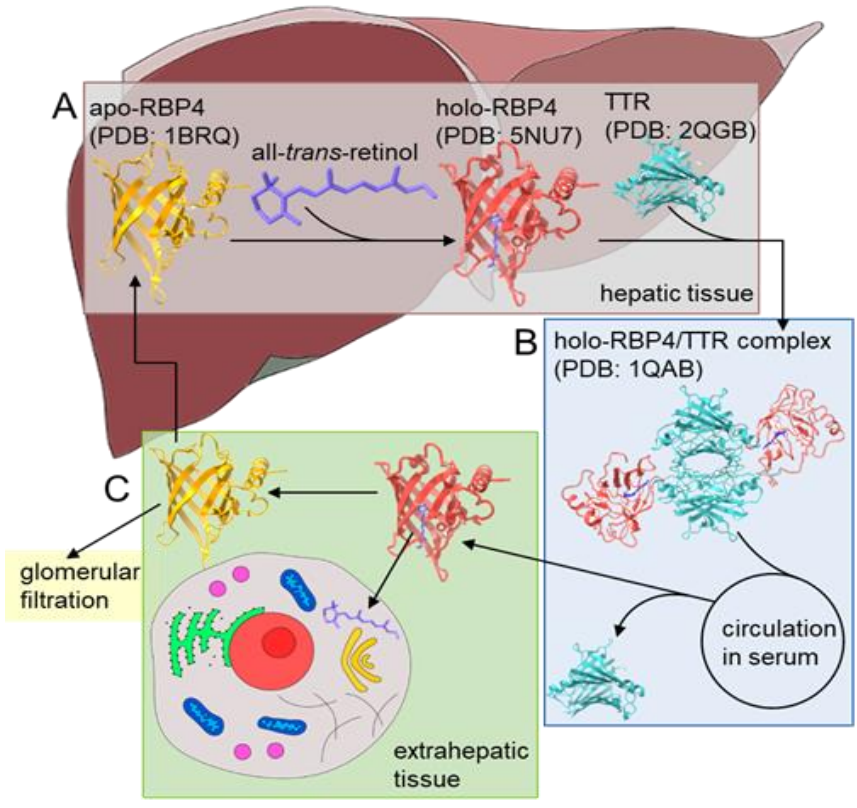

Scheme 1. RBP4-mediated transport of all-trans-retinol in blood serum from stores in the liver to extrahepatic peripheral tissue. A) Secretion of RBP4 from the rough endoplasmatic reticulum of hepatocytes to the blood serum. Binding of apoRBP4 with retinol and subsequent complexation of holo-RBP4 with transthyretin (TTR) forms a 1:1:1 holo-RBP4/TTR complex. Release into the serum is mediated by retinol availability, the conformational change upon retinol-binding is supposed to trigger the release. B) Delivery of retinol from hepatic retinoid stores to peripheral extrahepatic tissues during periods of inadequate vitamin A intake. Transport of the holo-RBP4/TTR complex in blood serum to target cells, there the complex is cleaved to release holo-RBP4. C) RBP4associated retinol uptake by peripheral tissue due to specific interactions of holo-RBP4 with RBP4-receptors on the surface of target cells. Free RBP4 is either cleared by glomerular filtration or returned to hepatic stores afterwards.

Since their introduction more than 20 years ago, nucleic acid aptamers have become serious alternatives, which offer considerable additional technical options making them increasingly attractive for different applications compared to antibodies or antibody derivatives. Aptamers are single-stranded oligonucleotides like RNA or single-stranded DNA (ssDNA) with surprising physical and chemical stability, high specificity and affinity in combination with low overall immunogenicity that can acquire different secondary and tertiary structures and are capable to bind defined targets. ${ }^{[18]}$ High-affinity aptamers can be evolved and isolated from large random sequence libraries. In this selection process performed completely in vitro called systematic evolution of ligands by exponential enrichment (SELEX) repeated rounds of target binding and PCR mediated amplification aptamers with binding affinity are successively enriched (Scheme 2). ${ }^{[19]}$ Additionally, to reduce the sequence space of the random nucleotide library, an initial elimination of nonspecific oligonucleotides by a counter selection using only the target carrier material prior to the selection process leads to a so called "counter library" with enhanced selectivity. Aptamers can be selected against an impressively different spectrum of target structures including proteins, ${ }^{[20]}$ whole cells, and microorganisms, ${ }^{[21,22]}$ small molecules and chemical compounds like metal ions[23]. They can not only be used in diverse fields such as diagnostics, ${ }^{[24]}$ biomarker discovery, ${ }^{[25]}$ imaging agents, ${ }^{[26]}$ drug delivery, ${ }^{[27]}$ and as pharmaceutical compounds in molecular therapy[28,29] but they are also attractive molecules for the construction of binding entities in technical devices like electronic biosensors ${ }^{[30]}$ on which predominantly antibodies or antibody derivatives have been used. ${ }^{[31-}$ 33]

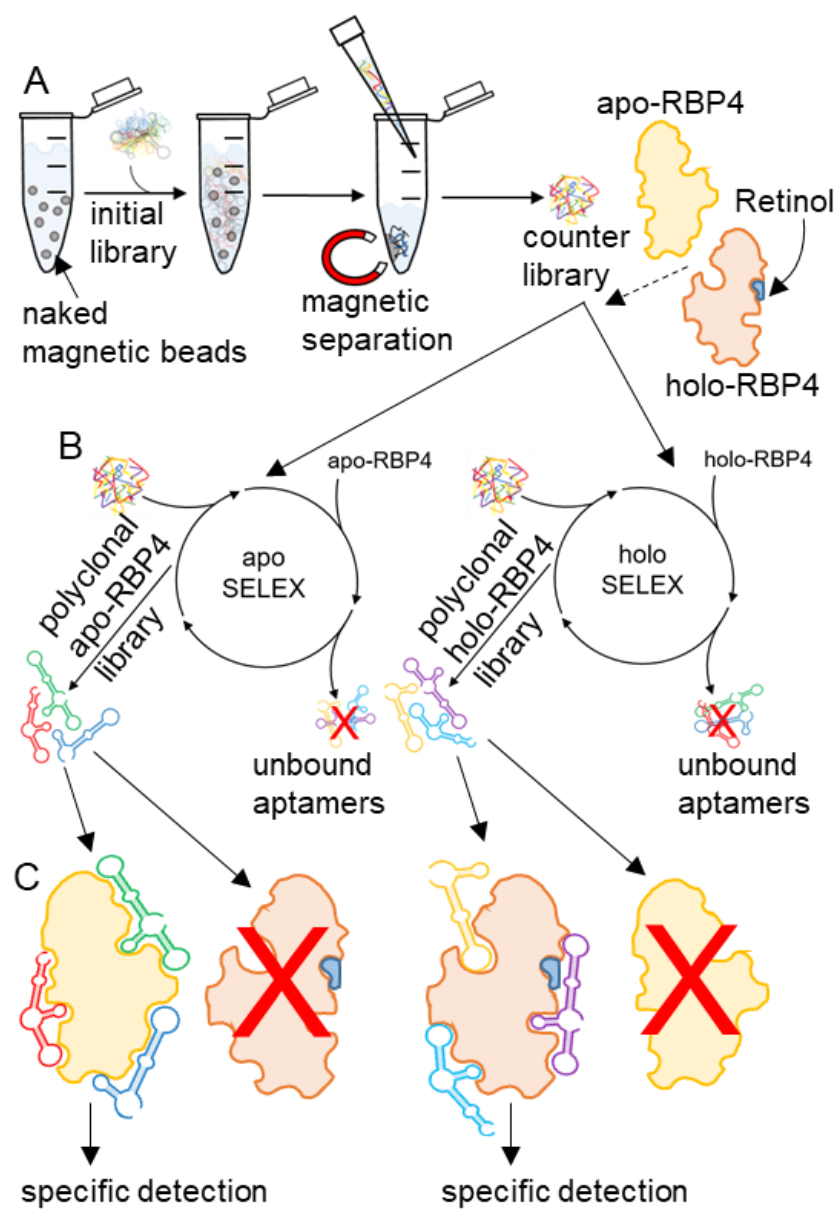

Scheme 2. SELEX based evolution of focused aptamer libraries for specific detection of apo- or holo-RBP4. A) Initial counter selection by incubation of an initial aptamer library ( $6 \times 10^{14}$ individual aptamers), containing 40 randomized nucleotides flanked by two primer binding sites, with naked Dynabeads ${ }^{\circledR} \mathrm{M}-280$ tosyl-activated resulting in an aptamer "counter library" with reduced amounts of aptamers with specificity against the naked beads. B) Selection of specific 
polyclonal aptamer libraries by SELEX against apo- or holoRBP4. Reduction of sequence diversity by incubating the counter selected aptamer library with the target proteins and aptamers exhibiting an adequate three-dimensional structure bind to the targets. The remaining unbound aptamers are subsequently removed, the bound aptamers are then eluted from the target proteins, amplified by ePCR and the undesired complementary strands are removed prior to the next SELEX round. C) Specific target detection by binding of focused aptamer libraries. Exclusive binding of polyclonal apo-RBP4 aptamers to apo-RBP4 and of holo-RBP4 aptamers to holoRBP4.

Recently, we have shown that as a significant simplification of the aptamer text book procedures, where the overall aim is to isolate and characterize individual aptamer sequences before they are used for applications, already focused polyclonal libraries can not only be used directly after sufficient enrichment, but can even outperform single aptamers. ${ }^{[22]}$ Thus, the use of focused polyclonal aptamer libraries can be expected to be advantageous due to higher precision based on the larger sequence space available for productive target recognition and increased performance also in sensor technologies and diagnostics. In order to specifically detect and quantify serum RBP4 such focused polyclonal aptamer libraries were evolved in an iterative SELEX process with recombinant purified target RBP4 proteins immobilized on magnetic particles in combination with fluorescence monitoring of the success of this molecular evolution process ("FluMagSELEX").[34] The aim was to create the possibility to differentiate between RBP4 isoforms (i. e. the apo- and holo- RBP4) two independent focused aptamer libraries were successfully evolved simultaneously against both apo- and holo-RBP4. The selective and precise quantification of RBP4 isoforms plays an important role in early diagnostics and in the reduction of either the level of serum apo- or holo-RBP4, as well as balancing impairments in RBP4 to retinol ratios. Exclusive specific binding of both polyclonal aptamer libraries to their dedicated targets was used for the subsequent development of aptamer-based apo-RBP4 and holoRBP4 biosensors.

Electrolyte-gated field effect transistor (EG-FET) devices have been used extensively in biosensing applications, for example to detect microRNA[35], $\mathrm{DNA}^{[36]}$ biomarkers for heart failure ${ }^{[37]}$, cancer ${ }^{[38]}$, biotin in the pM range ${ }^{[39]}$, and urea. ${ }^{[40]}$ The BST group has also recently developed a highly sensitive method of detecting the E7-protein for human papillomavirus implicated in carcinogenesis. ${ }^{[30]}$

One of the primary benefits of EG-FET sensing devices is that they do not require target labelling with fluorescent or redox probes. Both the sensor chips and the read-out equipment are low cost and therefore highly suitable for use in point-of-care settings and in low socioeconomic areas where expertise and laboratory environments are in short supply.

An EG-FET is based on the same principle as a metal oxide FET: a source and drain electrode are separated by a semiconducting channel (Scheme 3). By applying a voltage at the gate electrode, channel conductivity is changed and therefore the current flowing between the source and drain electrode. Biorecognition elements (antibodies, antigens, proteins or aptamers) can be deposited on the channel. When the target analyte binds, the change in dielectric layers at the interface between the channel and the electrolyte changes and as a result, the mobility of charge carriers in the channel is also changed. When the gate voltage is kept constant, binding events on the channel therefore change the source-drain current (IDS). Therefore, reduced graphene oxide field-effect transistors (rGOFETs, a sub-class of EG-FET devices) were functionalized with the selected polyclonal aptamer libraries and applied as an attractive sensing platform to monitor RBP4 levels. ${ }^{[30]}$
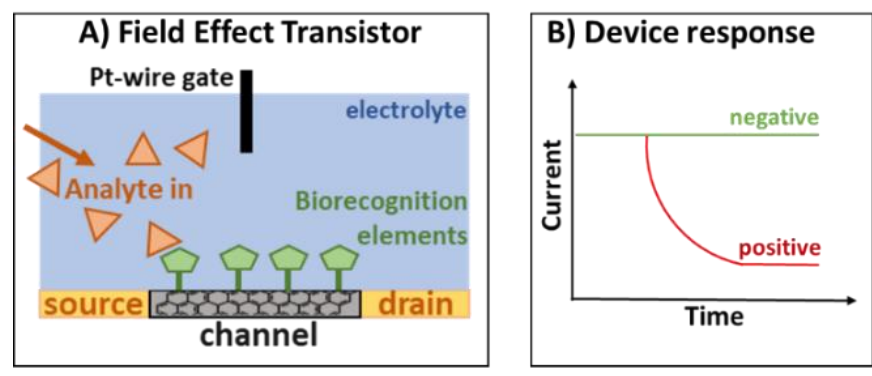

Scheme 3. A) Typical configuration of an EG-FET. B) Response of the device when there is no target analyte (green) and when the target analyte is present (red).

By replacing the platinum wire gate electrode, with planar gold, it is possible to combine optical and electronic sensing methods to achieve unparalleled real-time insight into biological interactions. ${ }^{[41]}$

\section{Results and Discussion}

Recombinant RBP4 production in E. coli and purification from cell extracts. Competent cells of the expression strain E. coli BL21 (DE3) were transformed freshly prior to RBP4 over-expression and grown for $5 \mathrm{~h}$ after induction with IPTG, and the cell growth was monitored photometrically. After its biotechnological production, cell harvest, and cell lysis almost no RBP4 was isolated from lysate supernatants but remained in the insoluble fraction (Figure S1A). This recombinant protein expressed in $E$. coli requires a denaturation and refolding process to be active and ready for retinol/retinal binding, as the formation of inclusion bodies was described for recombinant RBP4 
previously. ${ }^{[42]}$ It is not unusual that foreign proteins aggregate as inclusion bodies after high level overexpression in E. coli.[43] Several factors like a strong promotor system or a high target gene copy number favor a higher rate of recombinant protein expression leading to inclusion bodies formation.[44] Those can typically be dissolved under strongly denaturing conditions (e.g. urea) followed by incubation with the diluted denaturant in excess to enable protein refolding. ${ }^{[45,46]}$ Therefore, RBP4 was recovered according to Wang et al. from inclusion bodies as a soluble protein by dissolving the cell pellets in denaturation buffer containing $8 \mathrm{M}$ urea and subsequent refolding steps. It could be observed as a band with an apparent molecular mass of $21 \mathrm{kDa}$ (Figure S1A). After all refolding steps the resulting protein solution was submitted to size exclusion chromatography (SEC) for further purification. The collected fractions, assumed to contain purified RBP4, were analyzed by SDS-PAGE in Figure S1C, there single bands at a molecular mass of $\sim 21 \mathrm{kDa}$ represented RBP4. The corresponding protein concentrations (Figure S1B) were calculated by the ChromLab Software and a total yield of $\sim 12 \mathrm{mg} \mathrm{RBP4}$ was gained after urea extraction and purification from inclusion bodies harvested from $1 \mathrm{~L}$ culture.

Ligand binding properties and functionality of apoRBP4. The functionality of the purified RBP4 was further confirmed by determining its capacity to specifically bind increasing amounts of all-trans-retinal. Varying concentrations of retinal were added to a constant amount of the recombinant apo-RBP4. This mixture was incubated under exclusion of light and the quenching of intrinsic tryptophan fluorescence was monitored. The amount of apo-RBP4 in the reaction mixture decreased until only ligand-bound RBP4 was present after complexation with all-trans-retinal (Figure S1D). Interaction between RBP4 and retinal was confirmed, as the retinal quenched the intrinsic RBP4 tryptophan fluorescence due to energy transfer to the bound ligand. After binding, retinal is known to be incorporated into the binding cavity of the $\beta$-barrel of RBP4, where the polar groups remain solvent exposed. [2] High affinity binding of all-trans-retinal to E. coli-derived RBP4 was confirmed previously by spectral analysis. There, fluorescence quenching by binding increasing concentrations of retinal was monitored demonstrating the specific interaction at a single binding site. ${ }^{[47]}$ In order to select aptamer libraries against apo- or holo-RBP4, first immobilization of proteins on magnetic Dynabeads M-280 tosyl-activated was performed. Covalent coupling of the tosyl-groups on the surface of the magnetic beads with primary amino groups of apo-RBP4 was performed as described by the manufacturer. To verify the coating with apo-RBP4 or holo-RBP4, the intrinsic tryptophan fluorescence emission was monitored at $340 \mathrm{~nm}$ with an excitation at $280 \mathrm{~nm}$. Fluorescence was measured of apo-RBP4, apo- or holo-RBP4 coated beads, retinal, and naked magnetic beads (Figure S1E). Afterwards, to confirm retinal binding of holo-RBP4, emission was monitored at $490 \mathrm{~nm}$ with an excitation at $350 \mathrm{~nm}$. The immobilization could be verified, as apo-RBP4 coated beads showed similar tryptophan fluorescence as single apo-RBP4 and intrinsic holo-RBP4 was quenched after complexing apo-RBP4 immobilized on magnetic tosylbeads with retinal. In comparison, signals gained by holo-RBP4 coated beads and by unbound retinal were similar. Uncoated naked magnetic beads exhibited no signals after fluorescence measurements, as buffers were used purely in the coating process.

Evolution of aptamer libraries specific for RBP4 isoform and verification of their specificities. With the aim to develop a methodological tool for the specific quantification of serum apo- and holo-RBP4, polyclonal ssDNA aptamer libraries were evolved during an iterative FluMag-SELEX over a total of eight selection rounds. In order to differentiate between the RBP4 forms, two independent focused ssDNA aptamer libraries were selected in a simultaneous SELEX process against both RBP4 isoforms. These polyclonal aptamer libraries that bind to either apo-RBP4 or holoRBP4 were successfully selected from a random ssDNA library containing $\sim 6 \times 10^{14}$ individual aptamers with 40 randomized nucleotides flanked by two primer binding sites (23 nt each). The specificity was increased by early counter selection measures in which nonspecific oligonucleotides were eliminated by using empty (or "naked") magnetic beads prior to each selection round. During the individual rounds of the FluMag-SELEX, the aptamers were Cy5-labelled via labelled PCR primers and analyzed fluorescently afterwards. To verify the evolution progress defined amounts of aptamers $(10 \mathrm{pmol})$ from each SELEX round were incubated with apo- or holo-RBP4-coated magnetic beads after the selection process. The libraries against apo-RBP4 were incubated for $30 \mathrm{~min}$ at $25^{\circ} \mathrm{C}$ with apo-RBP4-coated magnetic beads and the holo-RBP4 libraries with holo-RBP4-coated beads and analyzed fluorescently after elution. The amount of eluted aptamers increased with each selection round indicating the progress of the enrichment process (Figure 1A). The resulting two focused polyclonal aptamer libraries were further analyzed after the final selection round for specific target binding. The affinity binding characteristics of both final aptamer libraries were examined by analysis using apo- or holo-RBP4 coated magnetic beads as well as naked beads. Therefore, $10 \mathrm{pmol}$ of round eight aptamer libraries were used for each of the RBP4 binding assays. The 
binding assays were performed using the apo-RBP4 library to specifically distinguish apo-RBP4 immobilized on magnetic beads from holo-RBP4 and the naked beads negative control. Then, the final polyclonal holoRBP4 library was analyzed similar in order to specifically detect holo-RBP4.

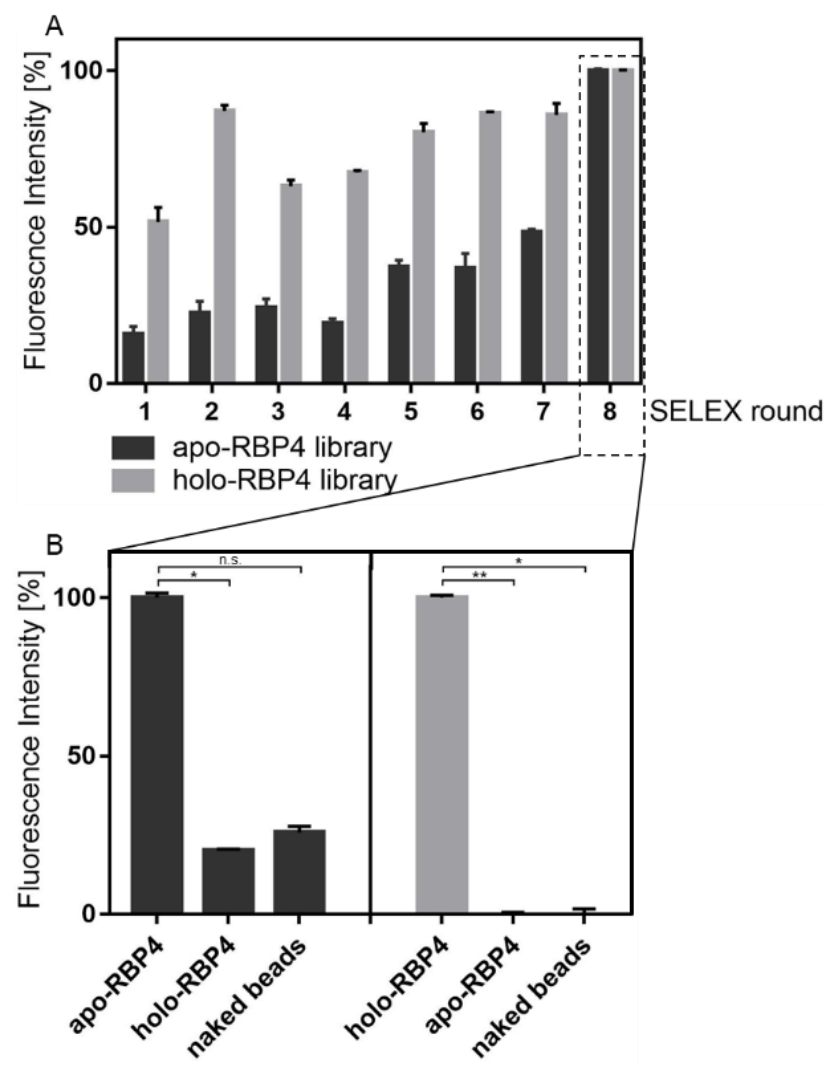

Figure 1. Characterization of specific RBP4 binding to Cy5labelled aptamer libraries. A) RBP4 ssDNA aptamer libraries enrichment. Black bars, increased binding of selected aptamers to apo-RBP4 during SELEX. Grey bars, increased binding of aptamers to holo-RBP4 during SELEX. The evolution progress was monitored using fluorescence measurement at an excitation of $635 \mathrm{~nm}$ with an emission at $670 \mathrm{~nm}$. B) Specificity analysis of the final polyclonal aptamer libraries. Binding of aptamers against apo-RBP4 or holoRBP4 to both apo- and holo-RBP4 as well as to naked Dynabeads $\AA$ M-280 tosyl-activated. All experiments were performed using $10 \mathrm{pmol}$ aptamers, $13.6 \mu \mathrm{g}$ apo- or holoRBP4 and $0.68 \mathrm{mg}$ magnetic beads, fluorescence was monitored at an excitation of $635 \mathrm{~nm}$ with an emission at $670 \mathrm{~nm}$. Error bars symbolize standard deviations of measurements conducted in triplicates. $P$ values $<0.05$ were considered significant. *denotes $\mathrm{P}<0.05,{ }^{*}<0.01$, n.s. not significant.

As expected, both aptamer libraries were confirmed to specifically and efficiently bind their dedicated targets. Only marginal signals were exhibited by the apo-RBP4 library for holo-RBP4 and magnetic beads (Figure 1B), but high specificity was observed for the holo-RBP4 aptamer library (Figure 1B). In addition, the highly selective target binding of both aptamer libraries was visualized using fluorescence microscopy. Therefore, $30 \mathrm{pmol}$ of each aptamer library were incubated for 30 min with RBP4-coated magnetic beads as well as with naked beads as negative controls. Afterwards, images were taken under transmitted light to visualize the magnetic beads and to detect target binding. Exclusive target binding of apo-RBP4 aptamers was observed visually, as only fluorescence signals were observed after the binding to apo-RBP4 coated magnetic beads (Figure 2A).

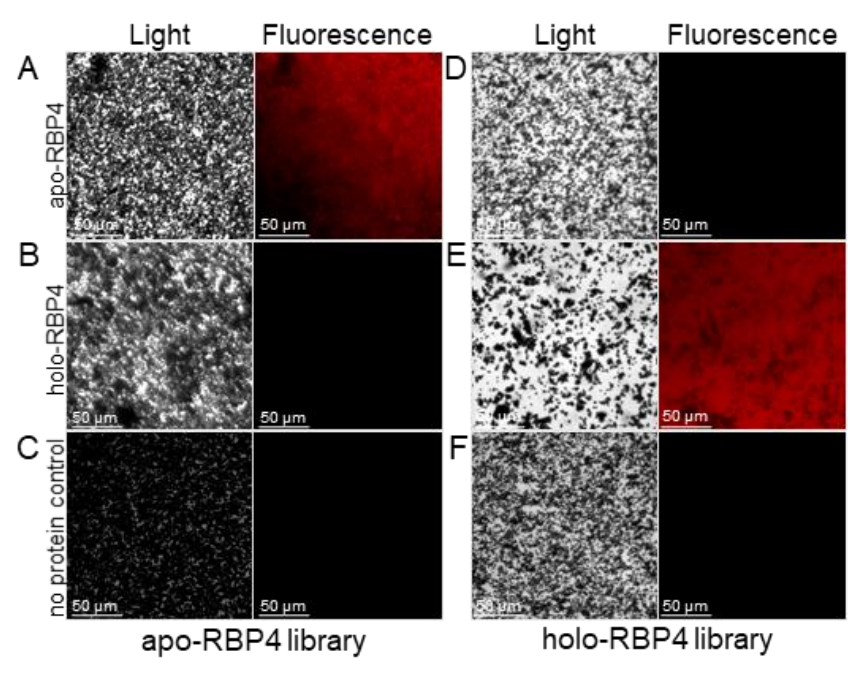

Figure 2. Fluorescence microscopic analysis of specific RBP4 binding to Cy5-labelled aptamer libraries. A) Binding of apoRBP4 aptamers to apo- and B) holo-RBP4 as well as to C) naked Dynabeads ${ }^{\circledR} \mathrm{M}-280$ tosyl-activated. D) Binding of holoRBP4 aptamers to apo- and E) holo-RBP4 as well as to F) naked Dynabeads $\AA$ M-280 tosyl-activated. All experiments were performed using $30 \mathrm{pmol}$ aptamers, $13.6 \mu \mathrm{g}$ apo- or holo-RBP4 and $0.68 \mathrm{mg}$ magnetic beads. Images were monitored using a Leica DMi8 coded (Leica Microsystems CMS GmbH, Wetzlar, Germany) at x40 magnitude under transmitted light and using the $Y 5$ filter (excitation: 590 $650 \mathrm{~nm}$ and emission: $662-738 \mathrm{~nm}$ ) for fluorescence imaging.

Contrarily, as expected beads coated with holo-RBP4 did not show any red fluorescence (Figure 2B) indicating the differentiation between both RBP4 forms by the apo-RBP4 library. Likewise, highly specific target recognition was observed for the holo-RBP4 aptamer library. Red fluorescence signals could be observed after the binding of these aptamers to their dedicated target holo-RBP4 (Figure 2D) and, as predicted, no signals were obtained after the incubation with apoRBP4-coated beads (Figure 2E). Moreover, both aptamer libraries exhibited no binding to the magnetic beads indicated by no fluorescence signals (Figure 2C, Figure 2F). These visual fluorescence signals additionally show the rapid and efficient labelling properties of both polyclonal aptamer libraries and furthermore the discriminating ability of them both between apo- and holo-RBP4. 


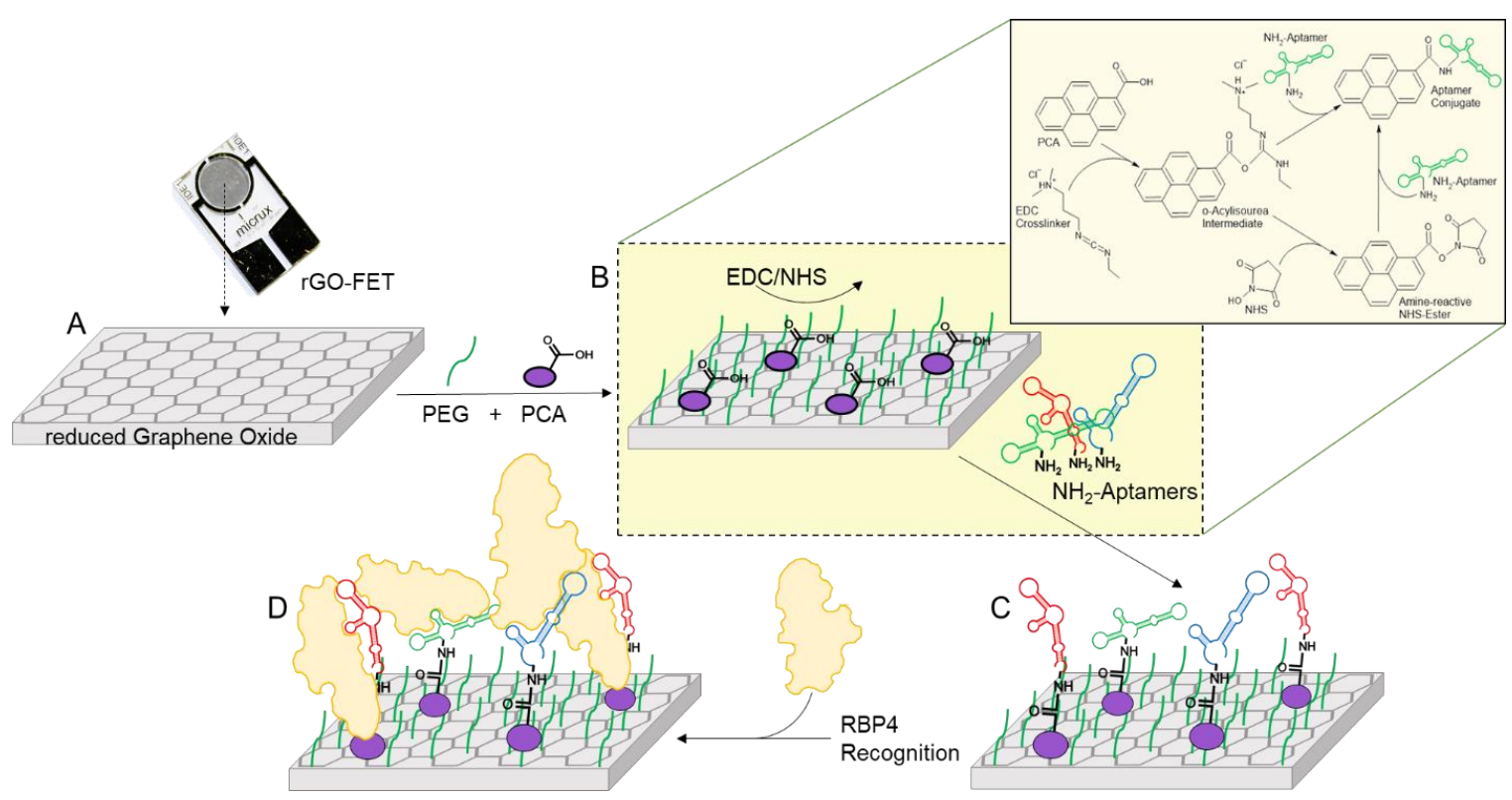

Scheme 4. Functionalization of rGO-FETs with polyclonal ssDNA aptamer libraries and specific apo- or holo-RBP4 detection. A) The rGO-FET were immersed into a mixture of PyPEG (PEG pre-conjugated with a PBSE) (500 $\mu \mathrm{M})$ and 1-pyrenecarboxylic acid (PCA, $50 \mu \mathrm{M}$, linker) in DMSO for $12 \mathrm{~h}$ at room temperature to obtain a 10:1 ratio of blocking and linking agents on the biosensor's surface. B/C) Apo- or holo-RBP4 aptamer library immobilization by first activating the carboxyl groups by immersion into a solution of EDC $(15 \mathrm{mM}) / \mathrm{NHS}(15 \mathrm{mM})$ in $150 \mathrm{mM}$ PBS solution for $30 \mathrm{~min}$, followed by covalent coupling of the 5'-NH2modified aptamer (100 nM in milliQ grade water for $40 \mathrm{~min}$ at $\left.25^{\circ} \mathrm{C}\right)$.D) Specific affinity recognition of either apo- or holo-RBP4 by the on rGO-FET immobilized polyclonal ssDNA aptamer libraries in electrical measurements.

Both the Dirac point shift and slope can change upon specific and unspecific binding, although the magnitude of the changes can vary between devices. The change in the $I_{D} V_{G}$ curve after addition of the target analyte is significantly larger than the shift after the addition of the non-target. To quantify the difference in sensor response in terms of the change in electron mobility of the device, we constructed a calibrated signal response graph in which we define the response signal $S=-\Delta \mathrm{I} / \mathrm{I}_{0}$, at that is, the change in the device current normalized by the current in the absence of analyte ( $\left.\mathrm{I}_{0}\right)$, at a given $V_{\text {GS }}$ : $-0.4 \mathrm{~V}$ (see Figure 3 for a graphic representation). It is important to note that some variation between devices exist and a device could only be characterized before and after analyte titration. Therefore, the gate voltage set point has to be decided prior to the measurement based on results from previous devices.

Typically, sensing with gFET devices is carried out by comparing shifts in $I_{D} V_{G}$ curves ${ }^{[35,48-50]}$, although realtime evaluation of binding by monitoring of source-drain current at fixed gate voltage during analyte addition has also been reported. ${ }^{[37,38,51]}$ The magnitude of the shifts in IDVG curves upon analyte binding enabled real time analyte detection in the sensor presented here.

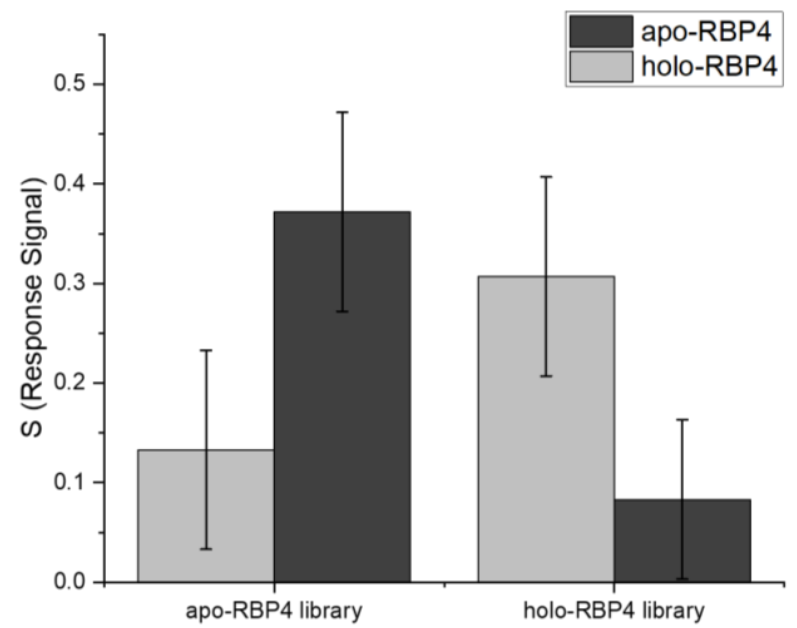

Figure 3. Difference in response signal of the sensors for the specific and non-specific interactions. The error bars are the standard deviation of at least three different IDVG curves. 

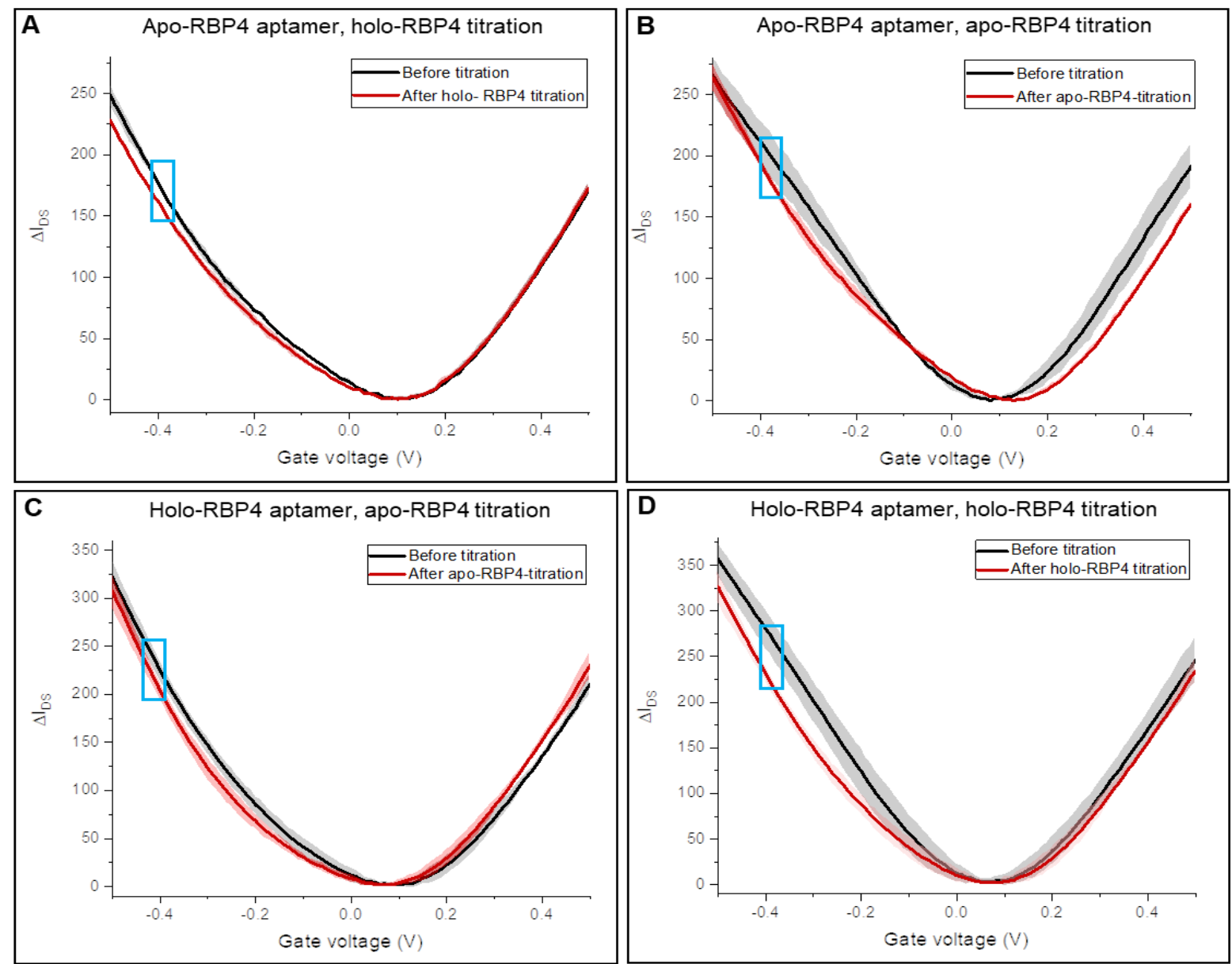

Figure 4. IDVG characteristics of transistors before and after the addition of the target analyte. The blue box shows the setpoint of the gate voltage during IDT measurements in sensing-mode. Each curve is an average of at least three measurements. The shadows of the lines show the standard deviation. IDVG curves were corrected for base line drift such that the Dirac point is set to a current of $0 \mu \mathrm{A}$.

The shift in $I_{D} V_{G}$ characteristics is due to changes in charge carrier mobility resulting from changes in the charge distribution at the channel-electrolyte interface. A change in the charges at the interface alters charge carrier mobility in the graphene and hence the sourcedrain current. ${ }^{[48]}$ Changes in charge distribution near the channel interface are expected to be mostly attributable to the binding of RBP4, which is positively charged at $\mathrm{pH}$ 7.4. Depending on the precise conformational changes and binding orientation (which are unknown at this stage), some changes could also arise from changes in the distance between the channel surface and the negatively charged phosphate backbone of the aptamers upon RBP4 binding.

A small shift in the shape and slope of the $I_{D} V_{G}$ curve occurred after the addition of the non-target analyte (Figure 4A and 4C), without any change in Dirac point. The small shift can be attributed to non-specific binding events of the analyte to the aptamers, or PEG groups on the surface. Addition of the target analyte resulted in a more pronounce shift in the $I_{D} V_{G}$ curve as well as a change in Dirac point and the slope of the curve, indicating stronger specific binding. The largest shift in the $I_{D} V_{G}$ curve could be seen in the negative gate voltage region between -0.3 and $-0.4 \mathrm{~V}$. Therefore, a gate voltage of $-0.4 \mathrm{~V}$ was chosen for $\mathrm{I}_{\mathrm{D}}(\mathrm{t})$ measurements.

In sensing-mode, constant source-drain and gate voltages are chosen (in this case $V_{D}=0.05 \mathrm{~V}, V_{G}=$ $0.4 \mathrm{~V}$ ) and the source-drain current is measured over time while the target analyte is added to the device. A binding event is indicated by either an increase or decrease in source-drain current. Titration curves of the transistor devices in sensing mode can be seen in Figure 5. The gFET devices were titrated with increasing concentrations of both isoforms of RBP4 to determine device selectivity and sensitivity. The limit of detection was found to be $100 \mathrm{pM}$ (Figure S2), which is around three orders or magnitude more sensitive than a previously reported aptamer-based RBP4 sensing method. ${ }^{[52]}$ However, at this concentration the change in signal intensity was too low for reliable reproducible sensing. However, these data suggest that by 
optimizing the functionalization density and method, sensitivity of the device could be significantly improved.

Figures $5 \mathrm{~A}$ and $5 \mathrm{D}$ show that while nonspecific binding occurred, binding events were much less pronounced than when adding the analyte for which the aptamer was selective. To our knowledge, this is the first report in which aptamer libraries were used for sensing purposes by depositing them on gFET devices. As the total concentration of RBP4 in human serum is in the range of 2-3 nM, we selected concentrations of $0.3,3$, 30 and $300 \mathrm{nM}$ to test the device. Real-time sourcedrain current traces of these concentrations are shown in Figure 5. While small nonspecific signals were observed, upon addition of the non-target protein, they were significantly smaller and often in the opposite direction to the specific binding events (see Figure $4 \mathrm{C}$ and 4D).

The sensor response to increasing analyte concentration is not proportional to analyte concentration, unlike previous reports of gFET-based. aptasensors. ${ }^{53]}$ There is a very strong response upon addition of 300 pM RBP4 (for both holo- and apo-RBP4 sensors), but a tenfold increase of analyte concentration fails to produce any response. A further tenfold analyte increase to $30 \mathrm{nM}$ produces a response, but the magnitude of the response is smaller than that observed when adding a 100-fold lower concentration. This could indicate that the aptamer libraries possess varying affinities for the analyte that is not continuously distributed but rather clusters around particular $K_{D}$ values. Once a particular subset of aptamers has been saturated, no further analyte binds until a concentration has been reached to saturate the next subset of aptamers.

Both aptamers are highly selective for the target analyte with only minor nonspecific binding at $300 \mathrm{pM}$. A small signal could be seen upon addition of 100 pM RBP4 (Figure S2), which corresponds to a theoretical detection limit of $2 \mathrm{ng} / \mathrm{mL}$ RBP4. However, given the typical concentration of 2-3 nM of RBP4 in blood serum, a low limit of detection is not as important as the ability to discriminate between loaded and unloaded RPB4 at physiologically relevant concentrations. Therefore, the maximum sensor response at $0.3 \mathrm{nM}$ is the ideal sensitivity range as it allows measurements in patient serum after 10 -fold dilution.

As the sensitivity of the device allows for dilution, interference of serum albumins with RBP4 detection should be reduced. Moreover, in this concentration range, sensor response is both very strong and highly selective. Therefore, a 10 -fold dilution of healthy patient sample should produce a pronounced signal with the holo-RBP4 aptamer library and little to no response in the device functionalized with the apo-library. Any increase in apo-RBP4 should produce a strong response in the opposite sensor configuration. A multiplexed sensor device can therefore carry out both the positive and negative control experiments and both healthy and unhealthy RBP4 concentrations will produce a significant response in one of the devices, prompting further clinical testing of the patient. Optimization of the sensor for measurements in blood plasma and multiplexing of the device will be the subject of a follow-up study. 

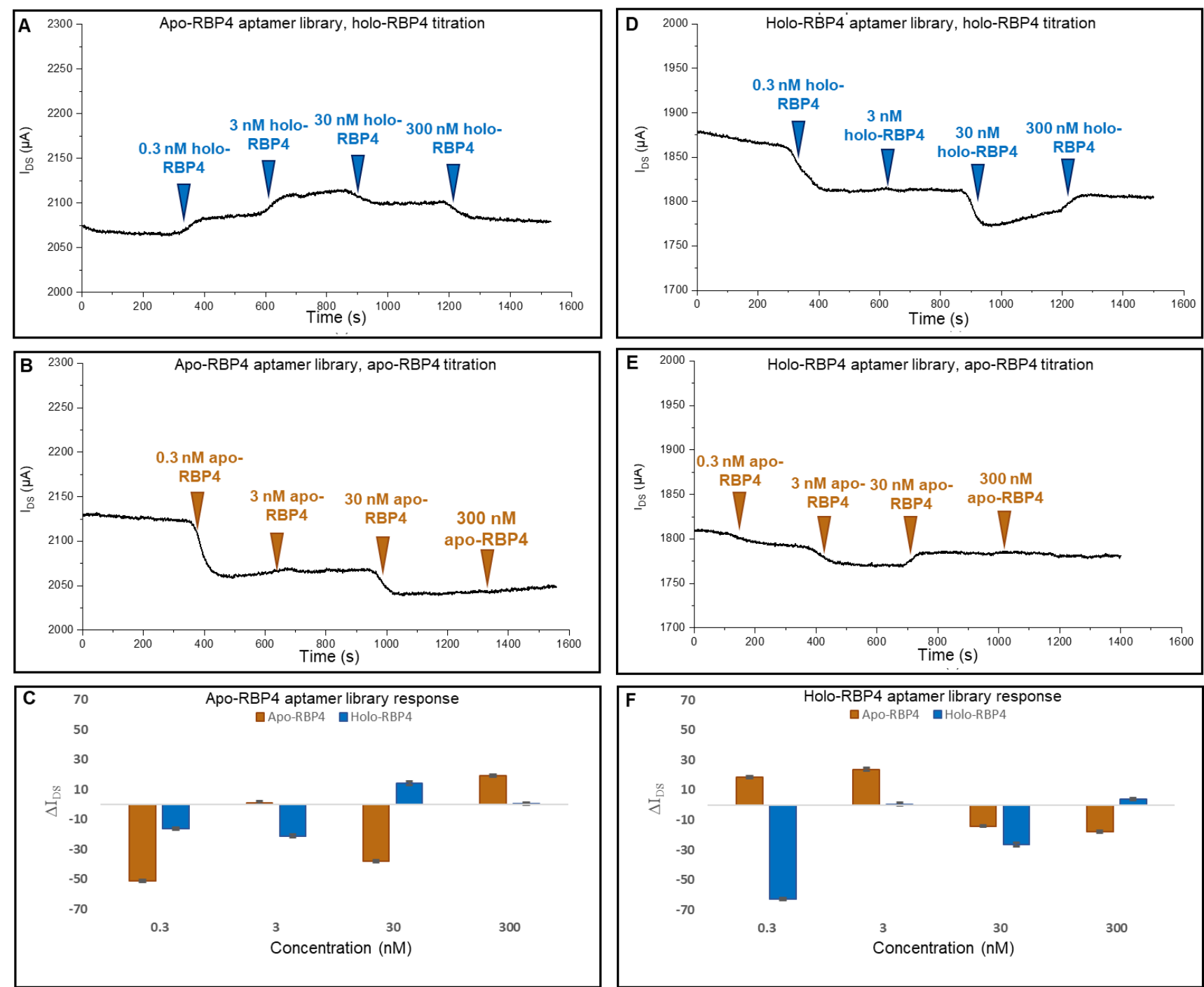

Figure 5. Titration curves of gFET-devices in sensing mode. A-C) Titration with holo-RBP4 (control) and apo-RBP4 in real time and $\triangle \mathrm{I}_{\text {DS }}$ for apo-aptamer library-functionalized gFETs, D-F) Titration with apo-RBP4 (control) and holo-RBP4 in real time and $\triangle \mathrm{l}_{\mathrm{DS}}$ for holo-aptamer library-functionalized gFETs. The bar graphs $\mathrm{C}$ and $\mathrm{F}$ depict the signal change at the equilibrium stage of the binding partners for each sensing library. 


\section{Conclusion}

The evolution of focused polyclonal aptamer libraries instead of single aptamers against target molecules serves as a promising strategy in diagnostics and sensor technology. Larger sequence spaces enable a distinct and specialized diversity of binding aptamers. Multiple target recognition can be achieved generating enhanced versatility and thus increased performance in sensing and quantification of target molecules. Moreover, the great biotechnological availability of polyclonal aptamer libraries contributes to the rapid, cost-effective and easy development of novel, highly specific electronic sensors for use as powerful tools in clinical diagnostics. By functionalizing the channel of an rGO-FET device, we were able to selectively discriminate between RBP4 isoforms at physiologically relevant concentrations between 0.3 and $30 \mathrm{nM}$. We believe that these results may open a route to develop biosensors for the fast and reliable measurement of the RBP4 isoform concentrations as a novel diagnostic marker.

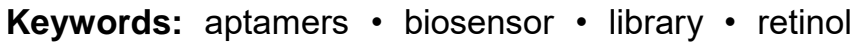
binding protein $4 \cdot$ SELEX

[1] E. Esteve, W. Ricart, J. M. Fernández-Real, Diabetes Care 2009, 32, S362 LP-S367.

[2] M. E. Newcomer, D. E. Ong, Biochim. Biophys. Acta - Protein Struct. Mol. Enzymol. 2000, 1482, 57-64.

[3] S. J. Thompson, A. Sargsyan, S. A. Lee, J. J. Yuen, J. Cai, R. Smalling, N. Ghyselinck, M. Mark, W. S. Blaner, T. E. Graham, Diabetes 2017, 66, 58-63.

[4] W. S. Blaner, Endocr. Rev. 1989, 10, 308-316.

[5] C. H. Chen, T. J. Hsieh, K. Der Lin, H. Y. Lin, M. Y. Lee, W. W. Hung, P. J. Hsiao, S. J. Shin, J. Biol. Chem. 2012, 287, 9694-9707.

[6] M. Kanai, A. Raz, D. S. Goodman, J. Clin. Invest. 1968, 47, 2025-2044.

[7] L. Quadro, L. Hamberger, V. Colantuoni, M. E. Gottesman, W. S. Blaner, Mol. Aspects Med. 2003, 24, 421-430.

[8] J. Raila, T. E. Willnow, F. J. Schweigert, J. Nutr. 2005, 135, 2512-2516.

[9] K. Kelly, S. Kashyap, V. O'Leary, J. Major, P. Schauer, J. Kirwan, Obesity (Silver Spring). 2009, 18, 663-666.

[10] C.-H. Chu, H.-C. Lam, J.-K. Lee, C.-C. Lu, C.C. Sun, H.-J. Cheng, M.-C. Wang, M.-J. Chuang, Endocr. J. 2011, 58, 841-847.
[11] Q. Yang, T. E. Graham, N. Mody, F. Preitner, O. D. Peroni, J. M. Zabolotny, K. Kotani, L. Quadro, B. B. Kahn, Nature 2005, 436, 356-362.

[12] T. E. Graham, Q. Yang, M. Blüher, A. Hammarstedt, T. P. Ciaraldi, R. R. Henry, C. J. Wason, A. Oberbach, P.-A. Jansson, U. Smith, B. B. Kahn, N. Engl. J. Med. 2006, 354, 2552-2563.

[13] S. K. Frey, B. Nagl, A. Henze, J. Raila, B. Schlosser, T. Berg, M. Tepel, W. Zidek, M. O. Weickert, A. F. H. Pfeiffer, F. J. Schweigert, Lipids Health Dis. 2008, 7, DOI 10.1186/1476-511X-7-29.

[14] H. Xu, G. T. Barnes, Q. Yang, G. Tan, D. Yang, C. J. Chou, J. Sole, A. Nichols, J. S. Ross, L. A. Tartaglia, H. Chen, J. Clin. Invest. 2003, 112, 18211830.

[15] J. M. Olefsky, C. K. Glass, Annu. Rev. Physiol. 2010, 72, 219-246.

[16] C. Erikstrup, O. H. Mortensen, A. R. Nielsen, C. P. Fischer, P. Plomgaard, A. M. Petersen, R. KroghMadsen, B. Lindegaard, J. G. Erhardt, H. Ullum, C. S. Benn, B. K. Pedersen, Diabetes, Obes. Metab. 2009, 11, 204-212.

[17] T. E. Graham, C. J. Wason, M. Blüher, B. B. Kahn, Diabetologia 2007, 50, 814-823.

[18] T. K. Sharma, J. G. Bruno, A. Dhiman, Biotechnol. Adv. 2017, 35, 275-301.

[19] C. Tuerk, L. Gold, Science 1990, 249, 505-510.

[20] P. Bayat, R. Nosrati, M. Alibolandi, H. Rafatpanah, K. Abnous, M. Khedri, M. Ramezani, Biochimie 2018, 154, 132-155.

[21] D. Kubiczek, N. Bodenberger, F. Rosenau, Antimicrob. Res. Nov. bioknowledge Educ. programs 2017, 6, 368-378.

[22] D. Kubiczek, H. Raber, N. Bodenberger, T. Oswald, M. Sahan, D. Mayer, S. Wiese, S. Stenger, T. Weil, F. Rosenau, Chem. - A Eur. J. 2020, 26, $14536-$ 14545.

[23] H. Qu, A. T. Csordas, J. Wang, S. S. Oh, M. S. Eisenstein, H. T. Soh, ACS Nano 2016, 10, 7558-7565.

[24] S. S. Sekhon, P. Kaur, Y. H. Kim, S. S. Sekhon, npj $2 D$ Mater. Appl. 2021, DOI 10.1038/s41699-02100202-7.

[25] A. Minopoli, B. Della Ventura, B. Lenyk, F. Gentile, J. A. Tanner, A. Offenhäusser, D. Mayer, R. Velotta, Nat. Commun. 2020, 11, 6134.

[26] J. Zhang, L. P. Smaga, N. S. R. Satyavolu, J. Chan, Y. Lu, J. Am. Chem. Soc. 2017, DOI 10.1021/jacs.7b07913. 
[27] T. Liang, Z. Yao, J. Ding, Q. Min, L. Jiang, J. J. Zhu, ACS Appl. Mater. Interfaces 2018, DOI 10.1021/acsami.8b14009.

[28] J. Zhou, J. Rossi, Nat. Rev. Drug Discov. 2017, DOl 10.1038/nrd.2016.199.

[29] G. Mahlknecht, R. Maron, M. Mancini, B. Schechter, M. Sela, Y. Yarden, Proc. Natl. Acad. Sci. 2013, 110, 8170-8175.

[30] P. Aspermair, V. Mishyn, J. Bintinger, $H$. Happy, K. Bagga, P. Subramanian, W. Knoll, R. Boukherroub, S. Szunerits, Anal. Bioanal. Chem. 2020, 7-11.

[31] Y. Chen, R. Ren, H. Pu, X. Guo, J. Chang, G. Zhou, S. Mao, M. Kron, J. Chen, Sci. Rep. 2017, DOI 10.1038/s41598-017-11387-7.

[32] S. Afsahi, M. B. Lerner, J. M. Goldstein, J. Lee, X. Tang, D. A. Bagarozzi, D. Pan, L. Locascio, A. Walker, F. Barron, B. R. Goldsmith, Biosens. Bioelectron. 2018, DOI 10.1016/j.bios.2017.08.051.

[33] G. Seo, G. Lee, M. J. Kim, S.-H. Baek, M. Choi, K. B. Ku, C.-S. Lee, S. Jun, D. Park, H. G. Kim, S.-J. Kim, J.-O. Lee, B. T. Kim, E. C. Park, S. II Kim, ACS Nano 2020, 14, 5135-5142.

[34] R. Stoltenburg, C. Reinemann, B. Strehlitz, Anal. Bioanal. Chem. 2005, 383, 83-91.

[35] B. Cai, L. Huang, H. Zhang, Z. Sun, Z. Zhang, G.-J. Zhang, Biosens. Bioelectron. 2015, 74, 329-334.

[36] S. Xu, S. Jiang, C. Zhang, W. Yue, Y. Zou, G. Wang, H. Liu, X. Zhang, M. Li, Z. Zhu, J. Wang, Appl. Surf. Sci. 2018, 427, 1114-1119.

[37] Y.-M. Lei, M.-M. Xiao, Y.-T. Li, L. Xu, H. Zhang, Z.-Y. Zhang, G.-J. Zhang, Biosens. Bioelectron. 2017, 91, 1-7.

[38] L. Zhou, H. Mao, C. Wu, L. Tang, Z. Wu, H. Sun, H. Zhang, H. Zhou, C. Jia, Q. Jin, X. Chen, J. Zhao, Biosens. Bioelectron. 2017, 87, 701-707.

[39] S. Wang, M. Z. Hossain, K. Shinozuka, N. Shimizu, S. Kitada, T. Suzuki, R. Ichige, A. Kuwana, H. Kobayashi, Biosens. Bioelectron. 2020, 165, 112363.

[40] T. Ono, Y. Kanai, K. Inoue, Y. Watanabe, S. Nakakita, T. Kawahara, Y. Suzuki, K. Matsumoto, Nano Lett. 2019, 19, 4004-4009.

[41] P. Aspermair, U. Ramach, C. Reiner-Rozman, S. Fossati, B. Lechner, S. E. Moya, O. Azzaroni, J. Dostalek, S. Szunerits, W. Knoll, J. Bintinger, J. Am. Chem. Soc. 2020, 142, 11709-11716.

[42] T. T. Y. Wang, K. C. Lewis, J. M. Phang, Gene 1993, 133, 291-294.
[43] A. Singh, V. Upadhyay, A. K. Upadhyay, S. M. Singh, A. K. Panda, Microb. Cell Fact. 2015, DOI 10.1186/s12934-015-0222-8.

[44] A. Mitraki, B. Fane, C. Haase-Pettingell, J. Sturtevant, J. King, Science (80-. ). 1991, DOI 10.1126/science.1648264.

[45] D. Shortle, M. S. Ackerman, Science (80-. ). 2001, 293, 487 LP - 489.

[46] Y. Xie, H. A. Lashuel, G. J. Miroy, S. Dikler, J. W. Kelly, Protein Expr. Purif. 1998, 14, 31-37.

[47] M. S. Levin, B. Locke, N. C. Yang, E. Li, J. I. Gordon, J. Biol. Chem. 1988, 263, 17715-17723.

[48] N. Nakatsuka, K.-A. Yang, J. M. Abendroth, K. M. Cheung, X. Xu, H. Yang, C. Zhao, B. Zhu, Y. S. Rim, Y. Yang, P. S. Weiss, M. N. Stojanović, A. M. Andrews, Science (80-. ). 2018, 362, 319 LP - 324.

[49] S. M. Majd, A. Salimi, F. Ghasemi, Biosens. Bioelectron. 2018, 105, 6-13.

[50] T.-Y. Chen, P. T. K. Loan, C.-L. Hsu, Y.-H. Lee, J. Tse-Wei Wang, K.-H. Wei, C.-T. Lin, L.-J. Li, Biosens. Bioelectron. 2013, 41, 103-109.

[51] B. Thakur, G. Zhou, J. Chang, H. Pu, B. Jin, X. Sui, X. Yuan, C.-H. Yang, M. Magruder, J. Chen, Biosens. Bioelectron. 2018, 110, 16-22.

[52] J. L. Su, B. S. Youn, W. P. Ji, J. H. Niazi, S. K. Yeon, B. G. Man, Anal. Chem. 2008, 80, 2867-2873.

[53] R. Stine, J. T. Robinson, P. E. Sheehan, C. R. Tamanaha, Adv. Mater. 2010, 22, 5297-5300. 